\title{
FACTORS ASSOCIATED WITH ATTENDANCE TO EARLY CHIDHOOD EDUCATION PROGRAMS IN MALAYSIA: RESULTS FROM THE NATIONAL HEALTH AND MORBIDITY SURVEY (MATERNAL AND CHILD HEALTH) 2016
}

\author{
Rajini Sooryanarayana ${ }^{1}$, Shubash Shander Ganapathy ${ }^{1}$, Rosliza Abdul Manaf ${ }^{2}$, Nik Mazlina Mohammad ${ }^{3}$, \\ Norazizah Ibrahim Wong ${ }^{1}$, Noor Ani Ahmad ${ }^{1}$, Chan Ying Ying ${ }^{1}$, Norhafizah Sahril ${ }^{1}$, Nor'Ain Abd Wahab ${ }^{1}$, \\ Nik Adilah Nik Shahein ${ }^{1}$ and Tahir Aris ${ }^{1}$ \\ ${ }^{1}$ Institute for Public Health, National Institutes of Health, Setia Alam, 40170 Shah Alam, Selangor, Ministry of Health \\ Malaysia \\ ${ }^{2}$ Faculty of Medicine and Health Sciences, Universiti Putra Malaysia, 43400 Seri Kembangan, Selangor, Malaysia \\ ${ }^{3}$ Kelana Jaya Health Clinic, Selangor State Health Department, 47301 Kelana Jaya, Ministry of Health Malaysia \\ Corresponding author: Rajini Sooryanarayana \\ Email: drrajini@moh.gov.my/
}

\section{ABSTRACT}

The National Health and Morbidity Survey (NHMS) 2016 was a nationwide cross-sectional survey focusing on maternal and child health. This study aimed to identify the prevalence of, and factors associated with early childhood educational program (ECE) attendance among children aged three to five years in Malaysia. Attendance to ECE has been shown to enhance children's development, yet there is no national information on ECE attendance in Malaysia, where attendance is not compulsory for children below five years. Parents were interviewed face-to-face utilising $a$ validated questionnaire from the UNICEF Multiple Indicator Cluster Survey using mobile devices. Multivariate logistic regression analysis was performed to identify factors associated with ECE attendance. Half (53.4\%) of children aged three to five years in Malaysia attend ECE. Older children aged four to five were six times more likely to attend ECE than children three to four years. Malaysian children of Chinese ethnicity were thrice as likely than ethnic Malays to attend ECE. Children with access to >3 children's books were twice more likely to attend ECE than those who had access to $<3$ books. Unemployed mothers were less likely to send their children to ECE than mothers employed in civil service. More awareness on the importance and benefits of ECE attendance on children's development is necessary so that younger children benefit from ECE.

Keywords: Early education, children, under-five, Malaysia, National Health and Morbidity Survey (NHMS).

\section{INTRODUCTION}

Malaysia is signatory to the Convention on the Rights of the Child $(C R C)^{1}$. Child rights under the CRC include rights to survival, development, protection and participation. A young child's psychosocial well-being and health are interdependent, having long lasting effects well into adulthood.

With the increasing influx of technology into daily lives coupled with lesser reading habits ${ }^{2}$, it is important to ensure that children are given the best stimulus for their developing brains in terms of toys, reading materials, and interaction with other children. Previous research highlights the importance of verbal interaction between children and their caregivers, as well as better quality of care, on their cognitive and social development. The advantages of early childhood education (ECE) on young children's development have been well documented, from intelligence quotient to social adjustment ${ }^{3}$.

In Malaysia, formal primary school attendance at age seven is almost universal because of mandatory enrolment ${ }^{4}$. Attendance at ECE programmes are important as they may influence children's readiness for primary school. The Malaysian national education system, through the Malaysian Education Act 1996 aims to provide equal opportunities for quality care and early education to pre-school aged children ${ }^{4}$. The National Education Policy 2012 focuses on the growth and development of children including physical, emotional, spiritual, intellectual and social development from birth to four years ${ }^{5}$.

ECE programmes offered by private and governmental providers have an organised learning structure, compared to commonly used baby-sitting and day-care services which generally do not. In Malaysia, a structured programme for children $<5$ years of age is not compulsory. However, various agencies from both government and private sectors have taken the initiative to provide these facilities.

A global study encompassing 63 countries found that $34 \%$ of three- and four-year olds attend ECE while $72 \%$ of children are engaged in various cognitive stimulation activities supporting learning by their caregivers. It also found that children from the richest backgrounds were 2.5 times more likely to attend ECE compared to children from the poorest households 6 . Previous research in Malaysia has shown that provisions for ECE were merely satisfactory, while no actual prevalence of those attending ECE were known, providing the impetus for the current survey. As ECE programmes are not compulsory for children 
$<5$ in Malaysia, this survey aimed to identify the extent of, and factors associated with ECE attendance among children aged 3-5 years.

\section{METHODOLOGY}

\section{Study design and sampling}

The National Health and Morbidity Survey (NHMS) is a population based, nationwide cross-sectional study. NHMS 2016 focused especially on maternal and child health $(\mathrm{MCH})$ and was conducted from February to May 2016 using stratified random sampling. It targeted community dwelling Malaysians, in non-institutionalised living quarters (LQ) in all 13 states and two Federal Territories in Malaysia from both urban and rural areas. The sample was selected from the database of live births registered with the National Department of Registration. Sample size was calculated based on the core objectives of the NHMS. To ensure national representativeness, stratified random sampling was done with states as the primary strata, while secondary strata was all districts within states. All mothers aged 15-49 years with children under five years within selected LQ were included.

Invitation letters, including consent forms, information sheets and pamphlets about the survey, were sent to the selected respondents in advance. Ministry of Health $(\mathrm{MOH})$ district community nurses visited the selected LQ to explain further about the survey and obtain written consent for the interview. Individuals were then invited to participate in the survey. The complete methodology of the NHMS 2016 is reported elsewhere ${ }^{7}$.

\section{Questionnaire}

This was based on the Multiple Indicator Cluster Survey (MICS) questionnaire developed by UNICEF $^{8}$, with permission. It comprised a household module as well as the $\mathrm{MCH}$ module. The questionnaire was administered in both Bahasa Melayu (Malay; the national language) and English. Validation for the local setting comprised a forward and backward translation from English to Malay and back which was done by content experts fluent in both languages. As Malaysia is a multi-ethnic multicultural society, the Malay version was pilot tested on all three major ethnicities in Malaysia; Malay, Chinese and Indians, of all education levels. Adequate face and content validity were thus established. The questionnaire was then used in the survey and administered as a face-to-face interview by trained interviewers using mobile devices. The ECE module was only administered to respondents with children aged 36-59 months at the time of interview.

\section{Variable definitions}

Household characteristics followed designated definitions used by the Department of Statistics Malaysia. Ethnicity was categorised using the three major ethnicities in Malaysia, the Malay, Chinese and Indians, besides Others comprising indigenous persons as well as local Sabahans and Sarawakians. Children's ethnicity was based on paternal ethnicity. Locality referred to either urban or rural areas, where an urban area is one with a population above 10,000 with at least $60 \%$ of its population aged above 15 years involved in non-agricultural activities?.

Only mothers aged $\geq 18$ years were eligible to be included in the ECE module. Mother's marital status was categorised into (i) married or cohabiting with partner, (ii) single, separated, divorced, or widowed. Maternal and paternal education levels were based on the Malaysian education system where: (i) those who had not attended any formal schooling or who had completed up to six years of primary school were categorised as having had no formal or primary education, (ii) those who had completed 11 years of formal schooling; secondary education, and (iii) those with diplomas or higher qualifications; tertiary education. Parental occupation was categorised into either working in the public sector, private sector, self-employed or unemployed, comprising those not working, stay at home mothers (housewives) or students. Household income was based on the reported pooled income of all family members in the household.

Attendance to ECE programmes was defined as usually attending any organised learning programme or early childhood education programme in the past seven days, either in private or government facilities, including kindergarten. It excluded baby-sitting or childminding, even if done in a day-care centre, unless the programme clearly included organised learning activities.

Information on various activities supporting early learning includes:

- parental engagement in the past three days in four or more of six cognitive stimulation activities,

- availability of reading materials appropriate for the child's age,

- playthings available to the child and

- daily screen time exposure.

For cognitive stimulation activities, the six activities are:

- involvement of adults in reading / looking at picture books together with children,

- playing together with various types of toys / games, 
- telling stories to the child,

- $\quad$ singing songs to or lullabies with the child,

- naming, counting or drawing things with the child, or

- going outdoors to play.

Any four answered 'yes' in the past three days was taken as a yes. Detailed information on the availability of reading materials appropriate for the children's age was asked about, specifically if the child had access to children's books or picture books, excluding newspapers, magazines, textbooks or adult books. Shared books were counted per child. Types of toys available to the child were asked about, and whether they had access to two or more different types of toys such as store-bought toys, homemade toys, and household objects used as playthings.

Daily screen time exposure included both active usage of tablets, smartphones, or computers, as well as passive exposure to television, where overall exposure of two hours or more a day was considered positive in children aged 3-5 years, following American Academy of Pediatrics guidelines $^{10}$.

\section{Ethical approval}

This survey was registered with and approved by the Malaysian Medical Research and Ethics Committee (NMRR-15-511-25359). All respondents were provided with an information sheet and a copy of the signed consent form. Thumb print impressions were taken in the presence of an impartial witness for illiterate respondents.

\section{Statistical analysis}

All statistical analyses were performed using SPSS software version $20.0^{11}$. Descriptive analyses were performed to describe the attendance of three to five-year olds to ECE programmes in the past 7 days. Sampling weights were applied to produce unbiased estimates. Logistic regression was performed for both univariable and multivariable analysis to identify factors associated with attendance to ECE programmes. The model was adjusted simultaneously for sociodemographic characteristics, parental engagement in $\geq 4$ cognitive stimulation activities in the past three days, children's books availability, playthings availability, and daily screen time exposure. Variables with $\mathrm{p}<0.25$ in the univariable analysis were included in the regression model.

\section{RESULTS}

\section{Baseline findings}

The response rate for the survey was $89.0 \%$. The prevalence of attendance to ECE programmes among children 3-5 years in Malaysia was $53.1 \%$ (95\% Cl: 50.44, 55.67) (Table 1). This may be extrapolated to 511,907 children of the 2,571,200 children $<5$ in Malaysia at the time of the survey.

Almost equal proportions of children attending ECE programmes were from urban and rural areas, as well as gender-wise. Children aged 48-59 months were twice as common to receive ECE compared to those 36-47 months old. Overall, attendance to ECE did not differ by mother's marital status. By ethnicity, the highest proportion of children aged 36-59 months attending an ECE programme was among Chinese (73.6\%), followed by Indians (59.6\%), Malays (51.4\%), and others (37.5\%). The highest proportion of children receiving ECE was reported among children with parents with higher education background, and higher earning. A higher proportion of working parents from public and private sectors appeared to send their children to ECE, compared to self-employed or unemployed parents. Almost equal proportions of children who attend ECE was seen among parents who did or did not engage in cognitive stimulation activities, as well as $\geq 2$, or $<2$ types of playthings being available to the child. Children with $<2$ hours exposure reported higher attendance to $E C E$, as did children with $\geq 3$ books availability.

\section{Factors associated with ECE attendance}

Bivariate analysis shows a significant association between age, ethnicity, parental educational level, mother's occupation, household income, screen time exposure and children's books availability towards attendance to ECE (Table 2 ).

From Table 3, univariable analysis showed that children of Chinese ethnicity, from households with higher income and with access to $>3$ books were more like to attend ECE. Children of other ethnicities, with mothers and fathers of lower educational levels, or unemployed, with $>2$ hours of screen time exposure daily were less likely to attend ECE.

In the multivariate analysis, fewer determinants were found significantly associated with ECE attendance. Older children aged 4-5 years were six times more likely to attend ECE than children 3-4 years (aOR 6.36, 95\% Cl: 5.00-8.11). Malaysians of Chinese ethnicity were thrice as likely (aOR 3.41, 95\% Cl: 2.22-5.22), while those of Indian ethnicity were twice as likely (aOR 2.25, 95\% Cl: 1.09-4.67) than Malays to attend ECE. Children with access to $>3$ children's books were twice more likely (aOR 1.79, 95\% Cl 1.37-2.34) to attend ECE. Unemployed mothers were significantly less likely to send their children to attend ECE than mothers in civil service (aOR 2.32, 95\% Cl 1.55-3.44). 
Table 1a: Early education programme attendance among Malaysian children under five, NHMS 2016

\begin{tabular}{|c|c|c|c|c|c|}
\hline \multirow[t]{2}{*}{ Characteristic } & \multirow[t]{2}{*}{$\mathbf{n}$} & \multirow[t]{2}{*}{$\%$} & \multicolumn{2}{|c|}{$95 \% \mathrm{Cl}$} & \multirow{2}{*}{$\begin{array}{l}\text { Estimated } \\
\text { population }\end{array}$} \\
\hline & & & Lower & Upper & \\
\hline $\begin{array}{l}\text { Early education program } \\
\text { attendance } \\
\text { Age (months) }\end{array}$ & 1,938 & 53.4 & 51.1 & 55.7 & 511,907 \\
\hline 48-59 months & 1,264 & 72.5 & 69.5 & 75.3 & 340,712 \\
\hline $36-47$ months & 674 & 35.0 & 31.8 & 38.3 & 171,195 \\
\hline \multicolumn{6}{|l|}{ Sex } \\
\hline Girls & 920 & 54.7 & 51.4 & 58.0 & 251,616 \\
\hline Boys & 1,018 & 52.1 & 48.9 & 55.3 & 260,291 \\
\hline \multicolumn{6}{|l|}{ Ethnicity } \\
\hline Chinese & 253 & 73.6 & 67.6 & 78.7 & 116,184 \\
\hline Indian & 81 & 59.4 & 45.4 & 72.0 & 31,325 \\
\hline Others & 156 & 37.5 & 32.5 & 42.9 & 54,549 \\
\hline Malay & 1,448 & 51.4 & 48.7 & 54.1 & 309,849 \\
\hline \multicolumn{6}{|l|}{ Strata } \\
\hline Urban & 1,159 & 54.0 & 50.9 & 57.1 & 330,103 \\
\hline Rural & 779 & 52.3 & 49.0 & 55.6 & 181,804 \\
\hline \multicolumn{6}{|l|}{ Mother's marital status } \\
\hline $\begin{array}{l}\text { Never married, separated, } \\
\text { divorced or widowed }\end{array}$ & 26 & 47.0 & 31.2 & 63.4 & 6,847 \\
\hline Married or cohabiting & 1,905 & 53.9 & 51.5 & 56.2 & 502,697 \\
\hline \multicolumn{6}{|l|}{ Mother's education level } \\
\hline No formal \& primary & 150 & 38.0 & 32.0 & 44.3 & 44,295 \\
\hline Secondary & 901 & 51.3 & 48.2 & 54.4 & 241,869 \\
\hline Tertiary & 875 & 62.0 & 58.0 & 65.8 & 222,577 \\
\hline \multicolumn{6}{|l|}{ Father's education level } \\
\hline No formal \& primary & 190 & 44.8 & 38.5 & 51.3 & 57,530 \\
\hline Secondary & 941 & 51.5 & 48.2 & 54.7 & 249,508 \\
\hline Tertiary & 739 & 62.6 & 58.3 & 66.6 & 183,348 \\
\hline \multicolumn{6}{|l|}{ Mother's occupation } \\
\hline Private & 396 & 61.7 & 56.5 & 66.7 & 131,654 \\
\hline Self-employed & 147 & 55.2 & 47.2 & 63.0 & 40,974 \\
\hline Unemployed & 718 & 44.8 & 41.5 & 48.1 & 200,485 \\
\hline Public & 669 & 64.2 & 59.5 & 68.6 & 136,199 \\
\hline \multicolumn{6}{|l|}{ Father's occupation } \\
\hline Private & 805 & 53.4 & 49.7 & 56.9 & 242,647 \\
\hline Self-employed & 498 & 54.2 & 50.0 & 58.4 & 140,527 \\
\hline Unemployed & 11 & 31.2 & 15.4 & 53.1 & 3,105 \\
\hline Public & 562 & 56.8 & 52.0 & 61.4 & 105,781 \\
\hline
\end{tabular}


Table 1b: Early education programme attendance among Malaysian children under five, NHMS 2016

\begin{tabular}{|c|c|c|c|c|c|}
\hline \multirow[t]{2}{*}{ Characteristic } & \multirow[t]{2}{*}{$\mathrm{n}$} & \multirow[t]{2}{*}{$\%$} & \multicolumn{2}{|c|}{$95 \% \mathrm{Cl}$} & \multirow{2}{*}{$\begin{array}{l}\text { Estimated } \\
\text { population }\end{array}$} \\
\hline & & & Upper & Lower & \\
\hline \multicolumn{6}{|l|}{ Household income (RM) } \\
\hline RM 5000 plus & 810 & 64.0 & 60.1 & 67.7 & 228,663 \\
\hline RM 4000-4999 & 214 & 53.6 & 46.2 & 61.0 & 50,235 \\
\hline RM 3000-3999 & 256 & 55.3 & 48.2 & 62.1 & 72,223 \\
\hline RM 2000-2999 & 264 & 46.6 & 41.2 & 52.0 & 66,261 \\
\hline RM 1000-1999 & 239 & 41.0 & 35.7 & 46.6 & 55,937 \\
\hline$<\mathrm{RM} 1000$ & 155 & 39.1 & 33.4 & 45.2 & 38,588 \\
\hline \multicolumn{6}{|c|}{$\begin{array}{l}\text { Parental engagement with } \\
\text { child }\end{array}$} \\
\hline 4 or more activities & 426 & 53.9 & 48.9 & 58.8 & 126,660 \\
\hline$<4$ activities & 1,512 & 53.2 & 50.6 & 55.8 & 385,247 \\
\hline \multicolumn{6}{|c|}{ Availability of playthings } \\
\hline 2 or more types & 1,503 & 53.2 & 50.5 & 55.9 & 372,416 \\
\hline$<2$ types & 435 & 53.8 & 49.2 & 58.4 & 139,491 \\
\hline \multicolumn{6}{|l|}{ Screen time } \\
\hline 2 or more hours & 370 & 47.7 & 43.6 & 51.8 & 123,501 \\
\hline$<2$ hours & 1,252 & 56.8 & 53.8 & 59.8 & 338,293 \\
\hline \multicolumn{6}{|c|}{ Children's books availability } \\
\hline 3 or more books & 1,653 & 58.2 & 55.5 & 60.8 & 435,869 \\
\hline$<3$ books & 284 & 36.2 & 32.1 & 40.6 & 76,023 \\
\hline
\end{tabular}


Table 2: Bivariate associations showing characteristics of children under five years who attend and do not attend early education programmes in Malaysia, NHMS 2016

\begin{tabular}{|c|c|c|c|c|c|c|}
\hline \multirow[t]{2}{*}{ Characteristic } & \multicolumn{2}{|c|}{ Attend program } & \multicolumn{2}{|c|}{ Do not attend } & \multirow[t]{2}{*}{ Total } & \multirow[t]{2}{*}{ p-value } \\
\hline & $\mathrm{n}$ & $\%$ & $\mathrm{n}$ & $\%$ & & \\
\hline Age (months) & & & & & & $<0.001^{*}$ \\
\hline 48-59 months & 1,264 & 72.5 & 458 & 27.5 & 1,722 & \\
\hline 36-47 months & 674 & 35.0 & 1,178 & 65.0 & 1,852 & \\
\hline Sex & & & & & & 0.271 \\
\hline Girls & 920 & 54.7 & 788 & 45.3 & 1,708 & \\
\hline Boys & 1,018 & 52.1 & 848 & 47.9 & 1,866 & \\
\hline Ethnicity & & & & & & $<0.001^{*}$ \\
\hline Chinese & 253 & 73.6 & 99 & 26.4 & 352 & \\
\hline Indian & 81 & 59.4 & 60 & 40.6 & 141 & \\
\hline Others & 156 & 37.5 & 268 & 62.5 & 424 & \\
\hline Malay & 1,448 & 51.4 & 1,209 & 48.6 & 2,657 & \\
\hline Strata & & & & & & 0.462 \\
\hline Urban & 1,159 & 54.0 & 951 & 46.0 & 2,110 & \\
\hline Rural & 779 & 52.3 & 685 & 47.7 & 1,464 & \\
\hline Mother's marital status & & & & & & 0.424 \\
\hline $\begin{array}{l}\text { Never married, separated, } \\
\text { divorced or widowed }\end{array}$ & 26 & 47.0 & 27 & 53.0 & 53 & \\
\hline Married or cohabiting & 1,905 & 53.9 & 1,584 & 46.1 & 3489 & \\
\hline Mother's education level & & & & & & $<0.001 *$ \\
\hline No formal \& primary & 150 & 38.0 & 253 & 62.0 & 403 & \\
\hline Secondary & 901 & 51.3 & 823 & 48.7 & 1,724 & \\
\hline Tertiary & 875 & 62.0 & 534 & 38.0 & 1,409 & \\
\hline Father's education level & & & & & & $<0.001^{*}$ \\
\hline No formal \& primary & 190 & 44.8 & 239 & 55.2 & 429 & \\
\hline Secondary & 941 & 51.5 & 853 & 48.5 & 1,794 & \\
\hline Tertiary & 739 & 62.6 & 446 & 37.4 & 1,185 & \\
\hline Mother's occupation & & & & & & $<0.001^{*}$ \\
\hline Private & 396 & 61.7 & 260 & 38.3 & 656 & \\
\hline Self-employed & 147 & 55.2 & 116 & 44.8 & 263 & \\
\hline Unemployed & 718 & 44.8 & 870 & 55.2 & 1,588 & \\
\hline Public & 669 & 64.2 & 365 & 35.8 & 1,034 & \\
\hline Father's occupation & & & & & & 0.131 \\
\hline Private & 805 & 53.4 & 687 & 46.6 & 1,492 & \\
\hline Self-employed & 498 & 54.2 & 439 & 45.8 & 937 & \\
\hline Unemployed & 11 & 31.2 & 22 & 68.8 & 33 & \\
\hline Public & 562 & 56.8 & 394 & 43.2 & 956 & \\
\hline Household income (RM) & & & & & & $<0.001^{*}$ \\
\hline RM 5000 plus & 810 & 64.0 & 462 & 36.0 & 1,272 & \\
\hline RM 4000-4999 & 214 & 53.6 & 148 & 46.4 & 362 & \\
\hline RM 3000-3999 & 256 & 55.3 & 217 & 44.7 & 473 & \\
\hline RM 2000-2999 & 264 & 46.6 & 291 & 53.4 & 555 & \\
\hline RM 1000-1999 & 239 & 41.0 & 297 & 59.0 & 536 & \\
\hline$<$ RM 1000 & 155 & 39.1 & 221 & 60.9 & 376 & \\
\hline Parental engagement with child & & & & & & 0.810 \\
\hline 4 or more activities & 426 & 53.9 & 1257 & 46.8 & 824 & \\
\hline$<4$ activities & 1,512 & 53.2 & 1238 & 46.8 & 2,750 & \\
\hline Availability of playthings & & & & & & 0.818 \\
\hline 2 or more types & 1,503 & 53.2 & 1,257 & 46.8 & 2,760 & \\
\hline$<2$ types & 435 & 53.8 & 379 & 46.2 & 814 & \\
\hline Screen time & & & & & & $<0.001^{*}$ \\
\hline 2 or more hours & 370 & 47.7 & 396 & 52.3 & 766 & \\
\hline$<2$ hours & 1,252 & 56.8 & 983 & 43.2 & 2,205 & \\
\hline Children's books availability & & & & & & $<0.001^{*}$ \\
\hline 3 or more books & 1,653 & 58.2 & 1,149 & 41.8 & 2,802 & \\
\hline$<3$ books & 284 & 36.2 & 486 & 63.8 & 770 & \\
\hline
\end{tabular}


Table 3a: Univariable and multivariable analysis of factors associated with early education programme attendance among Malaysian children under five, NHMS 2016

\begin{tabular}{|c|c|c|c|c|c|c|c|c|}
\hline \multirow[t]{2}{*}{ Characteristics } & \multirow[t]{2}{*}{ cOR } & \multirow[t]{2}{*}{ p-value } & \multicolumn{2}{|c|}{$95 \% \mathrm{Cl}$} & \multirow[t]{2}{*}{ aOR } & \multirow[t]{2}{*}{ p-value } & \multicolumn{2}{|c|}{$95 \% \mathrm{Cl}$} \\
\hline & & & Lower & Upper & & & Lower & Upper \\
\hline \multicolumn{9}{|l|}{ Age } \\
\hline 48-59 months & 4.886 & $<0.001^{*}$ & 3.987 & 5.989 & 6.363 & $<0.001^{* *}$ & 4.995 & 8.105 \\
\hline $36-47$ months & $\mathrm{R}$ & & & & $\mathrm{R}$ & & & \\
\hline \multicolumn{9}{|l|}{ Sex } \\
\hline Girls & 1.110 & 0.273 & 0.921 & 1.340 & - & & & \\
\hline Boys & $\mathrm{R}$ & & & & & & & \\
\hline \multicolumn{9}{|l|}{ Ethnicity } \\
\hline Chinese & 2.636 & $<0.001^{*}$ & 1.938 & 3.585 & 3.406 & $<0.001^{* *}$ & 2.224 & 5.217 \\
\hline Indian & 1.384 & 0.268 & 0.778 & 2.462 & 2.251 & $0.029 * *$ & 1.086 & 4.666 \\
\hline Others & 0.569 & $<0.001^{*}$ & 0.444 & 0.730 & 0.795 & 0.299 & 0.516 & 1.226 \\
\hline Malay & $\mathrm{R}$ & & & & $\mathrm{R}$ & & & \\
\hline \multicolumn{9}{|l|}{ Strata } \\
\hline Urban & 1.071 & 0.459 & 0.894 & 1.282 & - & & & \\
\hline Rural & $\mathrm{R}$ & & & & & & & \\
\hline \multicolumn{9}{|l|}{ Mother's marital status } \\
\hline $\begin{array}{l}\text { Never married, separated, divorced or } \\
\text { widowed }\end{array}$ & 0.759 & 0.427 & 0.385 & 1.497 & - & & & \\
\hline Married or cohabiting & $\mathrm{R}$ & & & & & & & \\
\hline \multicolumn{9}{|l|}{ Mother's education level } \\
\hline No formal \& primary & 0.375 & $<0.001^{*}$ & 0.275 & 0.512 & 0.708 & 0.170 & 0.432 & 1.160 \\
\hline Secondary & 0.646 & $<0.001^{*}$ & 0.524 & 0.796 & 0.935 & 0.714 & 0.651 & 1.341 \\
\hline Tertiary & $\mathrm{R}$ & & & & $\mathrm{R}$ & & & \\
\hline \multicolumn{9}{|l|}{ Father's education level } \\
\hline No formal \& primary & 0.486 & $0.001^{*}$ & 0.354 & 0.666 & 0.704 & 0.120 & 0.452 & 1.096 \\
\hline Secondary & 0.635 & $0.001^{*}$ & 0.509 & 0.792 & 0.776 & 0.115 & 0.566 & 1.064 \\
\hline Tertiary & $\mathrm{R}$ & & & & $\mathrm{R}$ & & & \\
\hline \multicolumn{9}{|l|}{ Mother's occupation } \\
\hline Private & 0.900 & 0.477 & 0.672 & 1.204 & 0.737 & 0.148 & 0.488 & 1.114 \\
\hline Self-employed & 0.689 & 0.054 & 0.472 & 1.006 & 0.638 & 0.077 & 0.387 & 1.050 \\
\hline Unemployed & 0.453 & $<0.001^{*}$ & 0.357 & 0.574 & 0.431 & $<0.001^{* *}$ & 0.288 & 0.646 \\
\hline Public & $\mathrm{R}$ & & & & $\mathrm{R}$ & & & \\
\hline \multicolumn{9}{|l|}{ Father's occupation } \\
\hline Private & 0.870 & 0.258 & 0.684 & 1.107 & 0.916 & 0.605 & 0.658 & 1.276 \\
\hline Self-employed & 0.903 & 0.431 & 0.699 & 1.165 & 1.179 & 0.364 & 0.826 & 1.682 \\
\hline Unemployed & 0.345 & $0.026^{*}$ & 0.136 & 0.879 & 0.292 & 0.090 & 0.071 & 1.210 \\
\hline Public & $\mathrm{R}$ & & & & $\mathrm{R}$ & & & \\
\hline
\end{tabular}




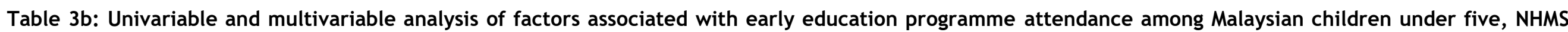
2016

\begin{tabular}{|c|c|c|c|c|}
\hline p-value & $95 \% \mathrm{Cl}$ & aOR & p-value & $95 \% \mathrm{Cl}$ \\
\hline & Lower & & & Lower \\
\hline
\end{tabular}

\section{Household income (RM)}

RM 5000 plus

\subsection{1}

$<0.001^{*}$

2.048

3.721

0.867

1.221

2.653

0.736

0.596

0.513

1.468

RM 4000-4999

1.800

$0.003^{*}$

1.321

2.794

0.771

0.309

0.407

1.329

\subsection{5}

$0.073^{*}$

0.953

1.887

0.802

0.292

0.475

1.250

RM 2000-2999

1.081

0.647

0.775

1.509

0.748

0.312

0.523

1.230

<RM 1000

$\mathrm{R}$

$\mathrm{R}$

Parental engagement with child

4 or more activities

1.028

0.821

1.288

0.679

$<4$ activities

$\mathrm{R}$

0.975

0.817

0.788

1.206

2 or more types

$\mathrm{R}$

Availability of books

3 or more books

2.449

$<0.001^{*}$

1.977

3.034

1.792

$<0.001^{* *}$

1.372

$<3$ books

$\mathrm{R}$

Screen time

2 or more hours

0.693

$0.001^{*}$

0.566

0.848

0.938

0.700

0.679

1.296 


\section{DISCUSSION}

Only half of Malaysian parents sent their children under-five years to ECE programmes, lower than neighbouring Thailand (84.0\%), Vietnam (71.3\%) and Singapore $(>95.0 \%)^{12-14}$. Of these, only half of parents who did so engaged actively with their child in various cognitive stimulation activities.

While Malaysia is a multi-ethnic and multicultural society, children of Chinese descent had the highest odds of attending ECE by 3.5 times more than ethnic Malays, who make up the largest ethnic group in Malaysia. Children of mothers who were not working were 2.3 times less likely to attend ECE compared to working mothers in the civil service.

Children with access to $\geq 3$ books were twice as likely to attend ECE than those with fewer books, a broad proxy measure for socioeconomic background, and literacy. Children from higher income households had higher odds of attending ECE. In comparison, developed countries reported either lower or higher prevalence of ECE attendance compared to Malaysia. Countries with lower prevalence ranging from $32 \%$ to $47 \%$ were Australia, New Zealand, United Kingdom and Sweden, while those higher than Malaysia were USA (60\%), Germany (65\% - 70\%), Japan and Hong Kong $(90 \%)$, as well as France $(100 \%)^{15}$. As parents' education has been shown to influence children's educational attainment ${ }^{16}$, we reviewed and found that Australia reported adult tertiary education of 49.3\%, New Zealand (43.4\%), United Kingdom (52.0\%), Sweden $(47.2 \%)$, whereas USA was $47.5 \%$, Japan $60.1 \%$, and France $44.0 \%$. These rates were spread across a continuum compared to the OECD average of $43.1 \%$, where tertiary education was defined as those having completed the highest level of education, by age group ${ }^{17}$.

Attendance to ECE is known to benefit children from different backgrounds with respect to cognitive, behavioural, schooling and health benefits, such as higher educational attainment, lower rates of crime involvement and substance abuse, which held true even in adulthood ${ }^{18}$. Children from poorer socioeconomic backgrounds with good ECE attendance rates benefit greatly, gaining more literacy skills than their counterparts from higher socioeconomic backgrounds during preschool and year one of primary schooling ${ }^{19}$.

Children of ethnic Chinese or Indians who are among the Malaysian minority ethnic groups having higher ECE programme attendance may be similar to studies showing that children of minority ethnicities have higher enrolment into ECE than the majority ethnicity ${ }^{20}$.

The civil service is the largest work sector in Malaysia, followed by the private sector. Those unemployed in this survey were stay-at-home mothers, housewives or students, from varying socioeconomic backgrounds. While some wellmeaning mothers may choose to both care for and educate their child, they may not know that ECE would boost their child's readiness for formal schooling, besides other benefits.

A recent global review suggested that integrating ECE within existing community programmes would enhance their sustainability in helping children achieve their developmental potential ${ }^{21}$. This was echoed in an Australian study of ECE centres in economically disadvantaged areas serving underprivileged children $<5$ years which provided high quality child education and care ${ }^{22}$.

When examining the factors that were significantly associated with ECE attendance, it appears that older age leads to six times higher odds of attending ECE compared to younger children. Recommendations to make ECE attendance compulsory for younger children should be considered. An Australian study however does recognise that 1-3 years of ECE prior to compulsory schooling may not have sufficient impact on children's well-being and development, until issues of availability and accessibility especially to vulnerable populations are addressed ${ }^{23}$. This is further supported by a Chinese study that emphasises strengthening of ECE among children from rural areas, its accessibility, availability, funding and management ${ }^{24}$.

Limitations of this study include the possibility of underreporting, recall bias in terms of parental engagement with the child, and social desirability bias. Subjective measures such as the quality of ECE was not ascertained. Furthermore, the sampling frame may have missed underserved populations with unregistered births as we used the sample of official births registered with the National Registration Department.

\section{CONCLUSION}

There is a need to increase awareness among Malaysian parents on the importance of attendance to ECE programmes that incorporate structured learning activities and not merely child-minding or babysitting, especially among younger children. Facilities available to all, including working mothers could be reviewed to help increase child enrolment. Creating awareness and educating parents on benefits of ECE would aid in achieving the Sustainable Development Goals.

\section{ACKNOWLEDGEMENTS}

The authors wish to thank the Director General of Health Malaysia for his permission to publish this study. The authors also wish to acknowledge the efforts of the staff of the Institute for Public Health, the various district health offices and the 
cooperation of all respondents who made this study possible.

\section{Funding}

This work was supported by the Ministry of Health Malaysia.

\section{Conflict of interest}

The authors declare no potential conflict of interest.

\section{Authors' contributions}

Rajini Sooryanarayana conceptualised the study, performed all statistical analyses and wrote the paper. Shubash Shander Ganapathy participated in study design and data analysis. Norazizah Ibrahim Wong, Noor Ani Ahmad and Tahir Aris supervised the data analysis and contributed to revising the paper. All authors helped plan the study and revise the manuscript.

\section{REFERENCES}

1. UNICEF. Convention on the Rights of the Child in Malaysia. 1995.

2. Shahriza Abdul Karim N, Hasan A. Reading habits and attitude in the digital age: Analysis of gender and academic program differences in Malaysia. The Electronic Library. 2007;25(3):285-298.

3. Duncan GJ. Modeling the impacts of child care quality on children's preschool cognitive development. Child development. 2003;74(5):14541475.

4. Attorney Generals Chambers Malaysia. Education Act 1996. 1996.

5. Ministry of Education Malaysia. National Education Policy. 2012.

6. McCoy DC, Salhi C, Yoshikawa H, Black M, Britto P, Fink G. Home-and center-based learning opportunities for preschoolers in low-and middleincome countries. Children and Youth Services Review. 2018;88:44-56.

7. Institute for Public Health (IPH). National Health and Morbidity Survey (NHMS 2016): Maternal and Child Health. Volume 1: Methodology and General Findings,2016. 120pp. 2016.

8. UNICEF. UNICEF MICS5 Tool. Questionnaire for children under five. 2015.
9. Department of Statistics Malaysia. Population Distribution and Basic Demographic Characteristics. 2010; http://www.statistics.gov.my/portal /download_Population/files/census2 010/Taburan_Penduduk_dan_Ciriciri_Asas_Demografi.pdf. Accessed 2 April 2012.

10. Strasburger VC, Hogan MJ, Mulligan DA, et al. Children, adolescents, and the media. Pediatrics. 2013;132(5):958-961.

11. IBM Corp. IBM SPSS Statistics for Windows, Version 20.0. Armonk, NY: IBM Corp. 2011.

12. National Statistical Office Thailand. Multiple Indicator Cluster Survey 2012, Thailand Final Report 2012.

13. General Statistical Office (GSO). Viet Nam Multiple Indicator Cluster Survey 2011,Final Report, 2011, Ha Noi, Viet Nam. 2011.

14. Ting TC. Policy developments in preschool education in Singapore: A focus on the key reforms of kindergarten education. International Journal of Child Care and Education Policy. 2007;1(1):35-43.

15. Boocock SS. Early childhood programs in other nations: Goals and outcomes. The future of children. 1995:94-114.

16. OECD. Tables A4.1 and A4.2. 2017; www.oecd.org/education/educationat-a-glance-19991487.htm Accessed 20 Nov 2017.

17. OECD. Population with tertiary education (indicator). 2017. Accessed 20 Nov 2017.

18. Reynolds AJ, Temple JA, Ou S-R, Arteaga IA, White BA. School-based early childhood education and age-28 well-being: Effects by timing, dosage, and subgroups. Science. 2011;333(6040):360-364.

19. Ready DD. Socioeconomic disadvantage, school attendance, and early cognitive development: The differential effects of school exposure. Sociology of Education. 2010;83(4):271-286.

20. Magnuson KA, Waldfogel J. Early childhood care and education: Effects on ethnic and racial gaps in school 
readiness. The future of children. 2005:169-196.

21. McCoy DC, Peet ED, Ezzati M, et al. Early childhood developmental status in low-and middle-income countries: national, regional, and global prevalence estimates using predictive modeling. PLoS medicine. 2016;13(6):e1002034.

22. 22. Ishimine K. Quality in early childhood education and care: a case study of disadvantage. The Australian Educational

Researcher. 2011;38(3):257-274.

23. Gilley T, Tayler C, Niklas F, Cloney D. Too late and not enough for some children: early childhood education and care (ECEC) program usage patterns in the years before school in Australia. International Journal of Child Care and Education Policy. 2015;9(1):9.

24. Li H, Yang W, Chen JJ. From 'Cinderella'to 'Beloved Princess': The evolution of early childhood education policy in China. International Journal of Child Care and Education Policy. 2016;10(1):2. 